\title{
LOCAL AND GLOBAL EXISTENCE FOR THE COUPLED NAVIER-STOKES-POISSON PROBLEM
}

$\mathrm{BY}$

\author{
DONATELLA DONATELLI
}

Dip. di Matematica Pura ed Applicata, Università degli Studi dell'Aquila, 67100 L'Aquila, Italy

\begin{abstract}
In this paper we investigate the Cauchy Problem for coupled NavierStokes-Poisson equation. The global existence of weak solutions in Sobolev framework is proved by using some compactification properties deduced from the Poisson equation.
\end{abstract}

1. Introduction. In this paper we deal with the existence of weak solutions for the coupled Navier-Stokes-Poisson problem. The interest for similar models is originated by the study of semiconductor devices. In the numerical simulation for semiconductor devices, the hydrodynamical models represent an acceptable compromise between accuracy and computational efficiency. Their common feature is the fact that the number of independent variables is reduced. Hydrodynamical models are obtained from the infinite hierarchy of the moment equations of the Boltzmann transport equation by suitable truncation procedures (sce for instance [6], [7], [18], [19]), and from these are derived the classical continuity equation, the momentum balance equation, and the energy balance equation ([2], [3], [10], [12], [13], [24]). In the case of a 1-D Euler-Poisson model, some results have been obtained in [16], [17]. In the model system considered here, we take into account the viscosity terms but we remove the equation regarding the energy (temperature). The removal of the energy balance is not completely justified from the physical point of view, since by adding viscosity it would be reasonable to include other physical quantities leading to effects of the same order. Unfortunately, the mathematical theory for the Navier-Stokes equation has been widely developed in the isoentropic case, but for the system including the energy balance equation, it is still at the initial state

Received October 31, 2001.

2000 Mathematics Subject Classification. Primary 76N10, 35Q30.

E-mail address: donatell@univaq.it 
(see [15] for details). In particular we consider the following system

$$
\left\{\begin{array}{l}
\frac{\partial n}{\partial t}+\operatorname{div}(n u)=0 \\
\frac{\partial n u}{\partial t}+\operatorname{div}(n u \Leftrightarrow u)+\nabla\left(a n^{\gamma}\right)-\mu \Delta u-\xi \nabla \operatorname{div} u=n \nabla \phi-n u \\
\Delta \phi=n-b(x)
\end{array}\right.
$$

in the region $\Omega \times(0, T), \Omega$ being a bounded connected open smooth domain in $\mathbb{R}^{N}$. The previous system is supplemented with the initial conditions

$$
n(x, 0)=n_{0}(x) . \quad n(x, 0) u(x, 0)=n_{0}(x) u_{0}(x), \quad \phi(x, 0)=\phi_{0}(x) .
$$

for any $x \in \Omega$ and the Dirichlet boundary condition

$$
u=0 \quad \text { on } \partial \Omega \times(0, T) .
$$

Here $n \geq 0$ denotes the electron density, $u \in \mathbb{R}^{N}$ the particle velocity. $\nabla \phi \in \mathbb{R}^{N}$ the electron field which is generated by the Coulomb force of the particles, the function $b=b(x)$ stands for the density of fixed positively charged background ions, $p=a n^{\gamma}, \gamma>1$ is the pressure density relation, and $j=n u$ will denote the electron current density.

In this paper we construct the weak solutions by using fixed point methods and compactness arguments; namely, we will consider the system

$$
\left\{\begin{array}{l}
\frac{\partial n}{\partial t}+\operatorname{div}(n u)=0 \\
\frac{\partial n u}{\partial t}+\operatorname{div}(n u \otimes u)+\nabla\left(a n^{\gamma}\right)-\mu \Delta u-\xi \nabla \operatorname{div} u=n \nabla \psi-n u \\
\Delta \phi=n-b(x)
\end{array}\right.
$$

with a given potential $\psi$. We will define the following fixed point map, from a suitable Banach space $X$ into itself, as follows. We say that

$$
A \psi=\phi \quad \text { if and only if } \quad \Delta \phi=n-b(x) .
$$

where $n$ solves the first two equations of (1.2) with prescribed $\psi$. At this point we need to show that the map $A$ is well-defined in suitable functional frameworks and will have some compactness properties to apply Schauder's fixed point theorem. The well-posedness of $A$ requires to show that the map $\psi \longrightarrow n(\psi)$ is well defined, namely that we can produce a solution to the problem (see [15])

$$
\left\{\begin{array}{l}
\frac{\partial n}{\partial t}+\operatorname{div}(n u)=0 \\
\frac{\partial n u}{\partial t}+\operatorname{div}(n u \otimes u)+\nabla\left(a n^{\urcorner}\right)-\mu \Delta u-\xi \nabla \operatorname{div} u=n \nabla \psi-n u .
\end{array}\right.
$$

Of course, the uniqueness problem to (1.3) is still a major open problem (see [15], Remark 7.7, pg. 180); hence we could produce, in principle, more than one map $A$. Hence the lack of uniqueness for (1.3) will reflect also on our results. Moreover, we will have another 
source of possible nonuniqueness which depends on the use of Schauder fixed point theory. A technical difficulty that one should keep in mind relies on the Lions theory, which assumes $\psi \in L^{\infty}\left(0 . T ; W^{1.2 \gamma /(\gamma-1)}(\Omega)\right)$, while in our case $\phi$ is reconstructed by means of div $j$ and this entails a loss of regularity with respect to $\psi$. We overcome this difficulty assuming some restriction on $\gamma$. The plan of the paper is as follows. In Sec. 2 we recall some of the theorems we are going to use later and we will define the kind of weak solutions we are going to build up. In Sec. 3, by using the theory of P. L. Lions [15], we investigate the existence of solutions for system (1.3). Then, in Sec. 4, we show that the map $A: X \longrightarrow X$ has a fixed point, which is our solution. Because of the nature of this problem we cannot apply a contractive-type theorem but we will use a Schauder-type fixed point theorem. In order to satisfy the requirements of this theorem we will get some restrictions on the possible values of $\gamma$ and on the time. Hence this will provide only a local solution for our problem. Later, by proving some uniform continuity in time of the local solutions, we will get the global existence.

\section{General Framework.}

2.1. Preliminary results. In this section we will recall the basic notations and theorems that will be used later on. We begin with the following version of the Ascoli Arzelà Theorem (see [22], pg. 135).

Theorem 2.1. Let $X$ be a compact metric space, $Y$ a Banach space, and $C(X ; Y)$ the Banach space of continuous functions from $X$ to $Y$ with the sup norm. A subset $H$ of $C(X ; Y)$ has compact closure in $C(X ; Y)$ if and only if

(i) $H$ is equicontinuous, and

(ii) for every $x \in X$ the set $H(x)=\{f(x) \mid f \in H\}$ has compact closure in $Y$.

Since in our system (1.1) we have a Poisson equation, we need the following elliptic regularity theorem [20]:

TheOrem 2.2. Consider the differential equation

$$
L u=\operatorname{div} A \nabla u=\operatorname{div} f+h, \quad \text { in } \Omega
$$

where $A=A(x)$ is a complex measurable matrix such that

$$
A=H+R
$$

where $H$ and $R$ are matrices such that the inequalities

$$
\begin{gathered}
\lambda|\xi|^{2} \leq(H \xi, \xi) \leq|\xi|^{2} \quad \lambda>0 \\
|R| \leq(1-\theta) \lambda \quad 0<\theta \leq 1
\end{gathered}
$$

hold for all relevant $x$ and $\xi$. If $\Omega$ is a bounded smooth domain, then (2.1) has a unique solution in $W_{0}^{1, p}(\Omega)$ for every complex vector field $f=f(x) \in L^{p}(\Omega)$ and every complex function $h=h(x) \in L^{r}(\Omega)$ with $r^{*} \geq p$. The solutions satisfy

$$
\|\operatorname{gradu}\|_{L^{p}(\Omega)} \leq C\|f\|_{L^{p}(\Omega)}+\|h\|_{L^{r}(\Omega)},
$$

where $C$ is a constant depending only on $\Omega, \theta \lambda, p$, and $r$.

We recall the following interpolation theorem ([4], pg. 310): 
TheOrem 2.3. Let $f \in L^{p}(\Omega) \cap W^{2 . r}(\Omega)$ with $1 \leq p \leq \infty$. Then $f \in W^{1 . q}(\Omega)$ where $\frac{1}{q}=\frac{1}{2}\left(\frac{1}{p}+\frac{1}{r}\right)$ and

$$
\|f\|_{W^{1, q}(\Omega)} \leq c\|f\|_{W^{* 2, \cdot(\Omega)}}^{1 / 2}\|f\|_{L^{\prime \prime}(\Omega)}^{1 / 2} .
$$

In particular, if $p=q=r$, then

$$
\|f\|_{W^{1, p}(\Omega)} \leq c\|f\|_{W^{\prime 2, p(\Omega)}}^{1 / 2}\|f\|_{L^{p(\Omega)}(1 / 2}^{1} .
$$

2.2. Statement of the problem. We will look for global weak solution of the following system

$$
\left\{\begin{array}{l}
\frac{\partial n}{\partial t}+\operatorname{div}(n u)=0 \\
\frac{\partial n u}{\partial t}+\operatorname{div}(n u \otimes u)+\nabla\left(a n^{\gamma}\right)-\mu \Delta u-\xi \nabla \operatorname{div} u=n \nabla \phi-n u \\
\Delta \phi=n-b(x)
\end{array}\right.
$$

in $\Omega \times(0, T), \Omega$ a bounded comnected open smooth domain in $\mathbb{R}^{N}$, endowed with the initial conditions

$$
\begin{aligned}
& n(x, 0)=n_{0}(x) \\
& n(x, 0) u(x, 0)=n_{0}(x) u_{0}(x)=j_{0}(x) \\
& \phi(x, 0)=\phi_{0}(x) \quad \text { for any } x \in \Omega
\end{aligned}
$$

and the Dirichlet boundary condition

$$
u=0 \quad \text { on } \partial \Omega(0, T) .
$$

Here the viscosity parameters satisfy $\mu>0, \mu+\xi>0$, moreover $a>0$ and the adiabatic constant $\gamma>1, n \geq 0, u \in \mathbb{R}^{N}, \phi \in \mathbb{R}$. We assume the following conditions hold:

(A.1) $\phi_{0} \in L^{1}\left(0, T ; W^{1.2 \gamma /(\gamma-1)}(\Omega)\right), \phi=\bar{\phi}$ on $\partial \Omega \times(0 . T), \bar{\phi}=0$,

(A.2) $b=b(x) \in L^{1}(\Omega)$.

(A.3) $n_{0} \geq 0 \quad$ a.e. in $\Omega, \quad n_{0} \in L^{1}(\Omega) \cap L^{\gamma}(\Omega)$,

(A.4) $j_{0} \in L^{2 \gamma /(\gamma+1)}(\Omega), \quad j_{0}=0 \quad$ a.e. on $\left\{n_{0}=0\right\}$.

(A.5) $\left|j_{0}\right|^{2} / n_{0} \in L^{1}(\Omega)$ defined to be 0 on $\left\{n_{0}=0\right\}$ and $n_{0} \neq 0$.

The conditions (A.1), (A.2), (A.3), (A.4), (A.5) are required to fit into the Lions framework [15]. At this stage, we are not imposing any further restrictions. We introduce here the notion of weak solution we are going to build and use.

Definition 2.4. By a weak solution of $(2.4)$, we mean $(n, u, \phi)$ satisfying $(2.4)$ in the sense of distributions such that

$$
\begin{gathered}
n \in L^{\infty}\left(0, T ; L^{\gamma}(\Omega)\right) \cap C\left([0, T] ; L^{p}(\Omega)\right) \quad \text { for } 1 \leq p<\gamma, n \geq 0 \text { a.e. } \\
\nabla u \in L^{2}\left(0, T ; L^{2}(\Omega)\right), \quad n|u|^{2} \in L^{\infty}\left(0, T ; L^{1}(\Omega)\right) \\
j=n u \in C\left([0, T], L^{2 \gamma /(\gamma+1)}(\Omega)-\text { weak }\right) .
\end{gathered}
$$




$$
\begin{array}{lr}
\phi \in L^{1}\left(0, T ; W^{1,2 \gamma /(\gamma-1)}(\Omega)\right) & \text { if } N \geq 2 \\
\phi \in L^{1}\left(0, T ; W^{1,2}(\Omega)\right) & \text { if } N=2 \text { and } \gamma>2 \\
\phi \in L^{1}\left(0, T ; W^{1,3 / 2}(\Omega)\right) & \text { if } N=3 \text { and } \gamma>6 .
\end{array}
$$

The above notion of weak solution is very natural in this frame since it simply follows from the energy identity which we will obtain in the next section.

3. Existence for the Navier-Stokes equations. This section is devoted to the analysis of the first two equations of the system (2.4), namely, the Navier-Stokes equations

$$
\begin{aligned}
& \frac{\partial n}{\partial t}+\operatorname{div}(n u)=0 \\
& \frac{\partial\left(n u_{i}\right)}{\partial t}+\operatorname{div}\left(n u u_{i}\right)+\partial_{i}\left(a n^{\gamma}\right)-\mu \Delta u_{i}-\xi \partial_{i} \operatorname{div} u=n \nabla \psi-n u_{i},
\end{aligned}
$$

$1 \leq i \leq N$, in $\Omega$, a bounded connected open smooth domain in $\mathbb{R}^{N}$, where $\mu>0$, $\mu+\xi>0, a>0$, and $\gamma>1$. Moreover, in this case $\psi=\psi(x, t)$ is fixed; it is a given function corresponding to the force terms on $\Omega \times(0, T)$ for some fixed $T \in(0, \infty)$. We assume the following Dirichlet boundary condition

$$
u=0 \quad \text { on } \partial \Omega \times(0, T)
$$

and the initial conditions

$$
\left.n\right|_{t=0}=n_{0} \quad \text { in } \Omega,\left.\quad n_{0} u\right|_{t=0}=j_{0} \quad \text { in } \Omega .
$$

In this section we report the main ideas from P. L. Lions's book [15] with some modifications in order to take into account the presence of the electric field and the collision term $n u$.

3.1. Estimates for the Navier-Stokes equations. We begin with some a priori estimates and compactness results.

3.1.1. A priori estimate. We specify better our initial condition; namely, we assume that $n_{0}, j_{0}$ satisfy

$$
\left\{\begin{array}{l}
n_{0} \geq 0 \quad \text { a.e. in } \Omega, \quad n_{0} \in L^{1}(\Omega) \cap L^{\gamma}(\Omega), \quad j_{0} \in L^{2 \gamma /(\gamma+1)}(\Omega), \\
j_{0}=0 \text { a.e. on }\left\{n_{0}=0\right\} \\
\left|j_{0}\right|^{2} / n_{0} \quad \text { defined to be } 0 \text { on }\left\{n_{0}=0\right\} \in L^{1}(\Omega) \text { and } n_{0} \neq 0
\end{array}\right.
$$

and for the moment we take $\psi \in L^{1}\left(0, T ; W^{1,2 \gamma /(\gamma-1)}(\Omega)\right)$. From Eq. (3.1) we get that $n$ remains non-negative for all $t>0$ and that $\int_{\Omega} n(t) d x$ is independent of $t \geq 0$. In addition, multiplying (3.2) by $u$, we deduce that

$$
\begin{aligned}
& n \frac{\partial|u|^{2} / 2}{\partial t}+n u \cdot \nabla \frac{|u|^{2}}{2}-\mu \Delta \frac{|u|^{2}}{2}-\xi \operatorname{div}(u \operatorname{div} u)+\mu|D u|^{2}+\xi(\operatorname{div} u)^{2} \\
& +\frac{\partial}{\partial t}\left(\frac{a}{\gamma-1} n^{\gamma}\right)+\operatorname{div}\left(u \frac{a \gamma}{\gamma-1} n^{\gamma}\right)=n u \cdot \nabla \psi-n u^{2}
\end{aligned}
$$


Integrating (3.6) with respect to $x$ and remembering the boundary condition on $u$, we deduce that

$$
\begin{aligned}
\frac{d}{d t} \int_{\Omega}\left\{n \frac{|u|^{2}}{2}+\frac{a}{\gamma-1} n^{\gamma}\right\} d x+\int_{\Omega} \mu|D u|^{2}+\xi(\operatorname{div} u)^{2} d x & =\int_{\Omega \Omega} n u \nabla \psi d x \\
& -\int_{\Omega} n u^{2} d x
\end{aligned}
$$

and obviously $\int_{\Omega}(\text { div } u)^{2} d x=\int_{\Omega} \partial_{i} u_{j} \partial_{j} u_{i} d x \leq \int_{\Omega}|D u|^{2} d x$. Since $\mu>0, \mu+\xi>0$, we deduce from (3.7) that we have for some $\nu>0$

$$
\frac{d}{d t} \int_{\Omega}\left\{n \frac{|u|^{2}}{2}+\frac{a}{\gamma-1} n^{\gamma}\right\} d x+\nu \int_{\Omega}|D u|^{2} \leq \int_{\Omega} n u \cdot \nabla \psi d x-\int_{\Omega} n u^{2} d x .
$$

Taking into account the condition on $\psi$, we have

$$
\begin{aligned}
\int_{\Omega} n u \nabla \psi d x & \leq\|n u\|_{L^{2 \gamma, \gamma+1}(\Omega)}\|\nabla \psi\|_{L^{2 \gamma / \gamma-1}(\Omega)} \\
& \leq\|\sqrt{n}\|_{L^{2 \Upsilon(\Omega)}(\Omega)}\|\sqrt{n} u\|_{L^{2}(\Omega)}\|\nabla \psi\|_{L^{2 \curlyvee / \gamma-1}(\Omega)} .
\end{aligned}
$$

Setting

$$
E(t)=\int_{\Omega}\left\{n \frac{|u|^{2}}{2}+n^{\gamma}\right\} d x
$$

and integrating on $t$, we have the following energy inequality

$$
E(t)+\nu \int_{0}^{t} \int_{\Omega}|D u|^{2} d t d x+\int_{0}^{t} \int_{\Omega} n u^{2} d t d x \leq E(0)+\int_{0}^{t} E(s) d s .
$$

Applying Gronwall's lemma. we get

$$
E(t) \leq c e^{c t}
$$

In this way we have proved the following

Theorem 3.1. Suppose $(n, u)$ is a weak solution of (3.1), (3.2), Suppose that (A.1), (A.2), (A.3), (A.4), (A.5) hold. Then $n \in L^{\infty}\left(0, T ; L^{\gamma}(\Omega)\right), \nabla u \in L^{2}\left(0 . T ; L^{2}(\Omega)\right)$, $n|u|^{2} \in L^{1}([0, T] \times \Omega), n u \in C\left([0 . T], L^{2 \gamma /(\gamma+1)}-\right.$ weak $)$.

Finally, we remark that since we have Dirichlet boundary conditions, we get also $u \in L^{2}\left(0, T ; H_{0}^{1}(\Omega)\right)$.

3.1.2. Compactness results. Existence results often follow from the analysis of the convergence of sequences of solutions and of the passage to the limits inside the equations. In this section we briefly report the results of P. L. Lions [15] on the analysis of the convergence and of the compactness of solutions. So we suppose to have a sequence of weak solutions $\left(n^{k}, u^{k}\right)$ of $(3.1)$ and $(3.2)$ with $\psi$ replaced by $\psi^{k}$ and some initial conditions

$$
\left.n^{k}\right|_{t=0}=n_{0}^{k} \quad \text { in } \Omega,\left.\quad n_{0}^{k} u^{k}\right|_{t=0}=j_{0}^{k} \quad \text { a.c in } \Omega .
$$

We always assume that $n_{0}^{k}, j_{0}^{k}$ satisfies (3.5) for all $k \geq 1$, and that $\left(n_{0}^{k}\right)_{k \geq 1}$ is bounded in $L^{1}(\Omega) \cap L^{\gamma}(\Omega),\left(j_{0}^{k}\right)_{k \geq 1}$ is bounded in $L^{2 \gamma /(\vartheta+1)}(\Omega)$, and $\left(\left|j_{0}^{k}\right|^{2} / n_{0}^{k}\right)_{k \geq 1}$ is bounded in $L^{1}(\Omega)$. By the energy inequality we can assume that

- $\left(n^{k}\right)_{k \geq 1}$ is bounded in $L^{\propto}\left(0, T ; L^{1} \cap L^{\gamma}(\Omega)\right)$,

- $\left(n^{k}\left|u^{\bar{k}}\right|^{2}\right)_{k \geq 1}$ is bounded in $L^{\infty}\left(0, T ; L^{1}(\Omega)\right)$. and 
- $\left(u^{k}\right)_{k \geq 1}$ is bounded in $L^{2}\left(0, T ; H_{0}^{1}(\Omega)\right)$.

Without loss of generality (extracting subsequences if necessary), we can assume that

- $n^{k} \rightarrow n$ weakly in $L^{\gamma}(\Omega \times(0, T))$ and $n \in L^{\infty}\left(0, T ; L^{1} \cap L^{\gamma}(\Omega)\right) n \geq 0$ a.e. $)$,

- $u^{k} \rightarrow u$ weakly in $L^{2}\left(0, T ; H_{0}^{1}(\Omega)\right)$,

- $\sqrt{n^{k}}-\overline{\sqrt{n}}$ weakly in $L^{2 \gamma}(\Omega \times(0, T))$,

- $\sqrt{n^{k}} u^{k} \rightarrow v$ weakly in $L^{2}(\Omega \times(0, T))$ and $v \in L^{\infty}\left(0, T ; L^{2}(\Omega)\right)$,

- $n^{k} u^{k} \rightarrow j$ weakly in $L^{2 \gamma /(\gamma+1)}(\Omega)$ and $j \in L^{\infty}\left(0, T ; L^{2 \gamma /(\gamma+1)}(\Omega)\right)$,

- $n^{k} u_{i}^{k} u_{j}^{k} \rightarrow e_{i j}$ in the sense of measures on $\Omega \times(0, T)$ and $e_{i j}$ is a bounded measure on $\Omega$ for almost all $t \in(0, T)$ which is bounded uniformly in $t \in(0, T)$,

- $n^{k} \nabla \psi^{k} \rightarrow \overline{n \nabla \psi}$ weakly in $L^{\gamma}(\Omega \times(0, T))$.

In order to get compactness it is necessary to assume some a priori bounds on $n^{k}$ that don't follow from the energy estimate. These bounds will be proved in the next section when we deal with the existence question. We thus assume that for some fixed $q \geq 2$, $q<\gamma, s>N / 2$

$\left(n^{k}\right)_{k \geq 1} \quad$ is bounded in $L^{q}\left(0, T ; L^{q}(K)\right) \cap L^{\infty}\left(0, T ; L^{s}(\Omega)\right) \quad K \subset \Omega$, compact set.

Now we can report the following theorem [15]:

TheOREM 3.2. (1) We always have: $v=\overline{\sqrt{n}} u, j=n u, e_{i j}=n u_{i} u_{j}$ a.e in $\Omega \times(0 . T)$ for all $1 \leq i, j \leq N$.

(2) If, in addition to the above assumptions, we assume that $n_{0}^{k}$ converges in $L^{1}(\Omega)$ to $n_{0}$, then $(n, u)$ is a weak solution of (3.1), (3.2) satisfying the initial conditions (3.4) and we have

$$
\begin{aligned}
n^{k} \longrightarrow n \quad & \text { in } C\left([0, T] ; L^{p}(\Omega)\right) \cap L^{r}(K \times(0, T)) \\
& \text { for all } 1 \leq p<s, 1 \leq r<q,
\end{aligned}
$$

$n^{k} u^{k} \longrightarrow n u$ in $L^{p}\left(0, T ; L^{r}(\Omega)\right)$ for all $1 \leq p<\infty, 1 \leq r<2 \gamma /(\gamma+1)$,

$u^{k} \longrightarrow u$ in $L^{p}(\Omega \times(0, T)) \cap\{n>0\}$ and in $L^{2}(\Omega \times(0 . T)) \cap\{n>\delta\}$

for all $1 \leq p<2$, for all $\delta>0$,

$$
\begin{aligned}
n^{k} u_{i}^{k} u_{j}^{k} \longrightarrow n u_{i} u_{j} \quad & \text { in } L^{p}\left(0, T ; L^{1}(\Omega)\right) \\
& \text { for all } 1 \leq p<\infty, \text { for all } 1 \leq i, j \leq N .
\end{aligned}
$$

One of the main tools in the proof of Theorem 3.2 is the use of renormalized solutions of (3.1), (3.2); namely, $u \in L^{2}\left(0, T ; H_{l o c}^{1}(\Omega)\right), n \in L_{l o c}^{2}(\Omega)$ satisfying (3.1), (3.2) are renormalized solutions of $(3.1),(3.2)$ if they satisfy

$$
\frac{\partial \beta(n)}{\partial t}+\operatorname{div}(u \beta(n))+(\operatorname{div} u)\left[\beta(n)-\beta^{\prime}(n) n\right]=0
$$

for any $\beta \in C^{1}([0, \infty))$ such that

$$
\exists C \geq 0, \quad \text { for all } t \geq 0, \quad\left|\beta^{\prime}(t)\right| \leq C(1+t)^{\alpha}
$$

with $\alpha=(q-2) / 2$, and also

$$
\beta\left(n^{k}\right)\left\{(\mu+\xi) d i v u^{k}-a\left(n^{k}\right)^{\gamma}\right\} \rightarrow \bar{\beta}\{(\mu+\xi) \operatorname{div} u-a \bar{p}\} \quad \text { in } \mathcal{D}^{\prime} .
$$


where $\bar{\beta}, \bar{p}$ are the weak limits of $\left(n^{k}\right)^{\hat{\gamma}} \cdot \beta\left(n^{k}\right)$ respectively. The use of the assumption (3.11) is also important in passing into the limit in the terms such as $n^{k}\left[u_{j}^{k} R_{i j}\right]\left(\dot{n}^{k} u_{i j}^{k}\right)$. where the operator $R_{i j}=(-\Delta)^{-1} \partial_{i j}$ is bounded in all $L^{r}$ spaces for $1<r<\infty$.

3.2. Existence results. In this section we are going to prove the existence of a solution $(n, u)$ of $(3.1),(3.2)$.

3.2.1. A priori bounds. We always assume that $n_{0}, j_{0}$ satisfy

$$
\left\{\begin{array}{l}
n_{0} \in L^{1}(\Omega) \cap L^{\gamma}(\Omega) \quad n_{0} \geq 0 \quad \text { a.e. in } \Omega . \quad n_{0} \neq 0 . \\
j_{0} \in L^{2 \gamma /(\gamma+1)}(\Omega) . \quad j_{0}=0 \text { a.e. on }\left\{n_{0}=0\right\} . \\
\left|j_{0}\right|^{2} / n_{0} \in L^{1}(\Omega) \quad \text { defined to be } 0 \text { on }\left\{n_{0}=0\right\} .
\end{array}\right.
$$

Following [15], in this section we take a more general hypothesis on $\psi$ :

$$
\nabla \psi \in L^{1}\left(0 . T: L^{2 \gamma /(\gamma-1)}(\Omega)\right)+L^{2 /(1+\alpha)}\left(0 . T: L^{r}(\Omega)\right) .
$$

where $\alpha=(2-\gamma)_{+}, \frac{1}{r}+\frac{1}{\gamma}\left(1-\frac{\alpha}{2}\right)+\frac{\alpha}{2}+\frac{1-\alpha}{q}=1$, and $q=\frac{2 N}{N-2}$ if $N \geq 3, q$ is arbitrary in $[2, \infty)$ if $N=2$. Then from the encrgy estimate we have the natural a priori bounds

$$
u \in L^{2}\left(0 . T: H_{0}^{1}(\Omega)\right), \quad n \in L^{\infty}\left(0, T: L^{\urcorner}(\Omega)\right) . \quad \||u|^{2} \in L^{1}([0 . T] \times \Omega) .
$$

In the last section the assumption (3.11) was important. Here. we want to derive this crucial a priori estimate on $n$.

Theorem 3.3. Let $(n, u)$ be a solution of (3.1), (3.2). We assume that $\nabla \psi \in L^{1}\left(0 . T: L^{3 / 2}\right)$ if $N=3$ and $\gamma>6$ and $\nabla \ell \in L^{1}\left(0 . T ; L^{2}\right)$ if $N=2$ and $\hat{\imath}>2$. We assume in addition that $n \in L^{\tilde{p}}(K \times(0, T))$ with $\bar{p}=\max (p, 2)$ and $p=\gamma+\frac{2}{N} \gamma-1, K$ being an arbitrary compact set in $\Omega$. Then, $n$ is bounded in $L^{p}\left(K^{*} \times(0 . T)\right)$ in terms of bounds on the data only.

RENARK 3.4. It is possible to provide some explanation for the exponent $p$ occuring in the above result. Indeed, on one hand $n \in L_{t}^{\infty}\left(L_{x}^{\gamma}\right)$, and on the other hand, if we simply expect $n^{2}$ and $n u \otimes u$ to have the same integrability, we deduce that $n^{\gamma-1}$ should belong to $L_{t}^{1}\left(L_{x}^{N /(N-2)}\right)$ since $u \in L_{t}^{2}\left(L_{x}^{N /(N-2)}\right)$ by Sobolev embeddings. These two bounds imply by interpolation that $n \in L_{x . t}^{p}$. with $p=\gamma+\frac{2}{N} \gamma-1$.

We will give only a sketch of the proof of Theorem 3.3, emphasizing the parts in which some modifications are needed because of the collision term $n u$.

Proof. There is nothing to prove if $\gamma \leq \frac{N}{2}$, since in that case, $p<\gamma-1$ and $n \in$ $L^{\infty}\left(0 . T ; L^{1} \cap L^{\gamma}\right)$. Therefore. in all that follows. we assime that $\gamma>\frac{N}{2}$. Since we are in Dirichlet boundary conditions we have to localize our problem. In order to do so, we introduce for any compact set $K \subset \Omega$ a cut-off function $\varphi \in C_{0}^{x}(\Omega)$ such that $0 \leq \varphi \leq 0$, $\varphi \equiv 1$ on $K$, and $\varphi \geq 0$ on $\Omega$. We then apply the divergence on Eq. (3.2):

$$
\frac{\partial}{\partial t} \operatorname{div}\left(n u_{i}\right)+\partial_{i} \partial_{j}\left(n u_{i} u_{j}\right)-(\mu+\xi) \Delta \operatorname{div} u+\Delta\left(a n^{\hat{\imath}}\right)=\operatorname{div}(n \nabla \psi)-\operatorname{div}\left(n u_{i}\right)
$$

Multiplying by $\varphi$ we obtain

$$
\begin{aligned}
\frac{\partial}{\partial t}\left(\varphi \operatorname{div}\left(n u_{i}\right)\right)+\partial_{i} \partial_{j}\left(\varphi n u_{i} u_{j}\right)-(\mu+\xi) \Delta(\varphi \operatorname{div} u)+\Delta\left(a \varphi n^{\gamma}\right)= & \operatorname{div}(\varphi n \nabla \psi) \\
& -\varphi \operatorname{div}\left(n u_{i}\right)+F
\end{aligned}
$$


where $F$ is given by

$$
\begin{aligned}
F & =\left(\partial_{i j} \varphi\right) n u_{i} u_{j}+2 \partial_{i} \varphi \partial_{j}\left(n u_{i} u\right)-(\mu+\xi) \Delta \varphi(\text { divu })+a \Delta \varphi n^{\gamma} \\
& -2(\mu+\xi) \nabla \varphi \cdot \nabla \text { divu }+2 a \nabla \varphi \cdot \nabla n^{\gamma}-n \nabla \psi \nabla \varphi .
\end{aligned}
$$

Now, applying the operator $\Delta^{-1}$ and multiplying the above equation by $n^{\theta}, \theta=p-\gamma$, we obtain

$$
\begin{aligned}
a \varphi n^{\gamma+\theta} & =\frac{\partial}{\partial_{t}}\left[n^{\theta}(-\Delta)^{-1}(\varphi \operatorname{div}(n u))\right]+\operatorname{div}\left[u n^{\theta}(-\Delta)^{-1}(\varphi \operatorname{div}(n u))\right]-n^{\theta}(-\Delta)^{-1} F \\
& -n^{\theta}(-\Delta)^{-1} \operatorname{div}(\varphi n \nabla \psi)+n^{\theta}(-\Delta)^{-1}(\varphi \operatorname{div}(n u))+(\mu+\xi) \varphi(\operatorname{divu}) n^{\theta} \\
& +n^{\theta}\left\{R_{i} R_{j}\left(\varphi n u_{i} u_{j}\right)-u \cdot \nabla(-\Delta)^{-1}(\varphi \operatorname{div}(n u))\right\} \\
& +(\theta-1)(\operatorname{divu}) n^{\theta}(-\Delta)^{-1}(\varphi \operatorname{div}(n u)),
\end{aligned}
$$

where $R_{i} R_{j}=(-\Delta)^{-1} \partial_{i} \partial_{j}$. We consider first when $N \geq 3$. If $N \geq 3$, we get an $L_{x, t}^{1}$ bound on $\varphi n^{\gamma+\theta}=\varphi n^{p}$ and thus on $\int_{K} n^{p} d x$. In fact, by integrating (3.19) over $\Omega \times(0, T)$, we deduce that

$$
\begin{aligned}
\int_{0}^{T} \int_{\Omega} n^{p} d x d t & \leq C+C \int_{\Omega} n^{\theta}(-\Delta)^{-1}(\varphi \operatorname{div}(n u)) d x \\
& +\int_{0}^{T} d t \int_{\Omega} d x|\operatorname{divu}| n^{\theta}\left(1+\left|(-\Delta)^{-1} d i v(n u)\right|\right) \\
& +C \int_{0}^{T} d t \int_{\Omega} d x n^{\theta}\left|R_{i} R_{j}\left(\varphi n u_{i} u_{j}\right)-u_{i} R_{i} R_{j}\left(\varphi n u_{j}\right)\right| \\
& +C \int_{0}^{T} d t \int_{\Omega} d x n^{\theta}\left|(-\Delta)^{-1} d i v(\varphi \nabla \psi)\right| \\
& +\int_{0}^{T} d t \int_{\Omega} d x n^{\theta}(-\Delta)^{-1} F+n^{\theta}(-\Delta)^{-1}(\varphi \operatorname{div}(n u)) .
\end{aligned}
$$

We focus our attention on the bound in $L^{1}(\Omega \times[0, T])$ of the new term

$$
n^{\theta}(-\Delta)^{-1}(\varphi \operatorname{div}(n u)) \text {. }
$$

Now we recall that $n^{\gamma}$ and $n|u|^{2}$ are bounded in $L^{\infty}\left(0, T ; L^{1}\right)$, while $D u$ is bounded in $L^{2}(\Omega \times(0, T))$ and $u$ (using Sobolev embeddings) is bounded in $L^{2}\left(0, T ; L^{2 N / N-2}(\Omega)\right)$. In particular, using Hölder inequality, $n u$ is bounded in $L^{\infty}\left(0, T ; L^{2 \gamma / \gamma+1}\right) \cap L^{2}\left(0, T ; L^{r}\right)$ where $\frac{1}{r}=\frac{N-2}{2 N}+\frac{1}{\gamma}$, and thus $n^{\theta}(-\Delta)^{-1}(\varphi \operatorname{div}(n u)) \in L^{\infty}\left(0, T ; L^{1} \cup L^{s}\right)$ where $\frac{1}{s}=$ $\frac{\theta}{\gamma}+\frac{\gamma+1}{2 \gamma}-\frac{1}{N}=\frac{1}{N}+\frac{1}{2}-\frac{1}{2 \gamma}<1$. The other terms are handled in the same way as in [15]. We deduce then from (3.19) that

$$
\int_{0}^{T} \int_{\Omega} \varphi n^{p} d x d t \leq C\left[1+\left(\int_{0}^{T} \int_{\Omega} n^{p}\left[|\nabla \varphi|^{p / \gamma}+|\Delta \varphi|^{p / \gamma}\right] d x d t\right)^{\gamma / p}\right]
$$

to get

$$
\int_{0}^{T} \int_{\Omega} \varphi n^{p} d x d t \leq C 1+\left(\int_{0}^{T} \int_{\Omega} \varphi n^{p} d x d t\right)^{\gamma / p} \leq C 1+T^{1-\frac{p}{\gamma}} \int_{0}^{T} \int_{\Omega} \varphi n^{\gamma} d x d t .
$$


Remembering that $\varphi \equiv 1$ on $K \subset \Omega$, a compact set, we obtain the desired estimate when $n \geq 3$.

Finally we consider the case when $N=2$. In that case we need to handle in a different way the terms $n^{\theta}\left\{R_{i} R_{j}\left(\varphi n u_{i} u_{j}\right)-u \cdot \nabla(-\Delta)^{-1}(\varphi \operatorname{div}(n u))\right\}$ and $\left[\operatorname{divun}^{\theta}(-\Delta)^{-1}(\varphi \operatorname{div}(n u))\right]$. After some calculation we have only to consider $n^{\theta}\left\{R_{i} R_{j}\left(\varphi n u_{i} u_{j}\right)-u_{i} R_{i} R_{j}\left(\varphi n u_{j}\right)\right\}$ and $\operatorname{divun}^{\theta}(-\Delta)^{-1}(\operatorname{div}(\varphi n u))$. In order to do so, we are going to multiply $(3.21)$ by $\varphi^{m}$ for some $m$ to be determined later on. Here we follow [15] exactly, but we point out that to estimate the term $\left\{R_{i} R_{j}\left(\varphi n u_{i} u_{j}\right)-u_{i} R_{i} R_{j}\left(\varphi n u_{j}\right)\right\}$, we use the fact that $u$ is bounded in $L^{2}\left(0, T ; H^{1}\right)$ and thus in $L^{2}(0, T ; B M O)$. Then, by the Coifman-Rochberg-Weiss commutator Theorem [5], we have for almost all $t \in[0, T]$

$$
\left\|R_{i} R_{j}\left(\varphi n u_{i} u_{j}\right)-u_{i} R_{i} R_{j}\left(\varphi n u_{j}\right)\right\|_{L^{2 p /(p+1)}} \leq C\|u\|_{B \Lambda I O}\|n u\|_{L^{2 p /(p+1)}},
$$

and we finally deduce that

$$
\left\|n^{\theta}\left\{R_{i} R_{j}\left(\varphi n u_{i} u_{j}\right)-u_{n}^{\theta} R_{i} R_{j}\left(\varphi n u_{j}\right)\right\}\right\|_{L_{x, t}^{2 p /(p+1)}} \leq C\|n\|_{L_{x, t}^{p}}^{1 / 2} .
$$

Hence, if we choose $m$ such that $m p / \theta \geq 1+m$ and $2 p \geq 1+m$, then we have

$$
\int_{0}^{T} d t \int_{\Omega} \varphi^{1+m} n^{p} d x \leq C\left(1+\left\|\varphi^{1+m / p} n\right\|_{L^{p / \theta}(\Omega \times[0, T])}^{\theta}\right)
$$

if we choose $m$ such that $m p / \theta \geq 1+m$. In conclusion, the proof is complete, provided we choose $m$ and $\varphi$ such that $\nabla \varphi^{m} \leq C$ holds and $1+m \leq 2 p, m p / \theta \geq 1+m$. In particular we may take $m=1$ and we conclude since $p \geq 1$ and $p=2 \gamma-1 \geq 2 \theta=2(\gamma-1)$.

3.2.2. Existence. We recall the notion of weak solution. We require $n$ and $u$ to satisfy (3.1), (3.2) and

$$
\begin{gathered}
n \in L^{\infty}\left(0, T ; L^{1} \cap L^{\gamma}(\Omega)\right) \cap C\left([0, T], L^{r}(\Omega)\right) \quad \text { for all } 1 \leq r<\gamma, n \geq 0 \text { a.e. } \\
u \in L^{2}\left(0, T ; H_{0}^{1}(\Omega)\right), \quad n|u|^{2} \in L^{\infty}\left(0, T ; L^{1}(\Omega)\right) \\
n u \in C\left([0, T], L^{2 \gamma /(\gamma+1)}(\Omega)-\text { weak }\right) .
\end{gathered}
$$

Finally, as in Theorem 3.3, we assume that $\nabla \psi \in L^{1}\left(0, T ; L^{3 / 2}\right)$ if $N=3$ and $\gamma>6$ and $\nabla \psi \in L^{1}\left(0, T ; L^{2}\right)$ if $N=2$ and $\gamma>2$.

TheOREM 3.5. Under the above conditions, and if we assume that $\gamma \geq \frac{3}{2}$ if $N=2, \gamma \geq \frac{9}{5}$ if $N=3$, and $\gamma>\frac{N}{2}$ if $N \geq 4$, there exists a solution $(n, u)$ of (3.1) and (3.2) satisfying the initial conditions (3.4) and such that $n \in L^{p}(K \times(0, T))$ for any compact set $K \subset \Omega$, where $p=\gamma+\frac{2}{N}-1$. In addition $(n, u)$ satisfies the following energy inequality for almost all $t \in[0, T]$ :

$$
\begin{aligned}
\int_{\Omega} n \frac{|u|^{2}}{2} & +\frac{a}{\gamma-1} n^{\gamma} d x+\int_{0}^{t} d s \int_{\Omega} \mu|D u|^{2}+\xi(\text { divu })^{2}+n u^{2} d x \\
& \leq \int_{\Omega} \frac{1}{2} \frac{\left|j_{0}\right|^{2}}{n_{0}}+\frac{a}{\gamma-1} n_{0}^{\gamma} d x+\int_{0}^{t} d s \int_{\Omega} n u \nabla \psi d x .
\end{aligned}
$$

Moreover, for any $R>0$, if

$$
\left\|n_{0}\right\|_{L^{\gamma}(\Omega)} \leq R \quad\left\|j_{0}\right\|_{L^{2 \gamma /(\gamma+1)}(\Omega)} \leq R \quad\|\psi\|_{W^{1,2 \gamma /(\gamma-1)(\Omega)}} \leq R,
$$


then there exists $c(R)>0$ such that

$$
\|n(\cdot, t)\|_{L^{\infty}\left(0, T ; L^{\gamma}(\Omega)\right)} \leq c(R) \quad\|j(\cdot, t)\|_{L^{\infty}\left(0, T ; L^{2 \gamma /(\gamma+1)}(\Omega)\right)} \leq c(R) .
$$

REMARK 3.6. The uniqueness of solutions, the regularity of solutions, and the fact that this energy inequality (3.26) might be an equality are outstanding open questions.

REMARK 3.7. It is possible to prove that the momentum $j=n u$ of the Navier-Stokes equation has actually some time regularity with values into $L^{2 \gamma /(\gamma+1)}$ endowed with its strong topology (see [8] for details).

The proof of Theorem (3.5) will be made "backwards": if Eq. B approximates Eq. A, we first explain how solutions of A can be obtained from solutions of B before explaining how to obtain the latter via solutions of C. Here we report for completeness the first step of the proof from [15].

Proof. We consider a "periodic box" $\widetilde{\Omega}=\prod_{i=1}^{N}\left(a_{i}, b_{i}\right)$ such that $\bar{\Omega} \subset \widetilde{\Omega}$; without loss of generality, we may take $a_{i}=0$ and set $T_{i}=b_{i}-a_{i}$ for $1 \leq i \leq N$. Then we introduce $p \in C^{\infty}\left(\mathbb{R}^{N}\right)$, periodic in each $x_{i}$ of period $T_{i}$ for each $i \in\{1, \ldots, N\}$, such that $p \equiv 0$ in $\bar{\Omega}, p>0$ in $\overline{\widetilde{\Omega}}-\bar{\Omega}$. We then consider the following problem in $\mathbb{R}^{N} \times(0, T)$, where the solution $\left(n^{\varepsilon}, u^{\varepsilon}\right)$ is required to be periodic in $x_{i}$ of period $T_{i}$ for each $i$ and $\varepsilon \in(0,1]$ :

$$
\left\{\begin{array}{l}
\frac{\partial n^{\varepsilon}}{\partial t}+\operatorname{div}\left(n^{\varepsilon} u^{\varepsilon}\right)=0 \quad n^{\varepsilon} \geq 0 \\
\frac{\partial\left(n^{\varepsilon} u^{\varepsilon}\right)}{\partial t}+\operatorname{div}\left(n^{\varepsilon} u \otimes u^{\varepsilon}\right)+a \nabla\left(n^{\varepsilon}\right)^{\gamma}-\mu \Delta u^{\varepsilon}-\xi \nabla \operatorname{div} u^{\varepsilon}+\frac{1}{\varepsilon} p u^{\varepsilon}=n^{\varepsilon} \nabla \widetilde{\psi}-n^{\varepsilon} u^{\varepsilon},
\end{array}\right.
$$

where $\tilde{\psi}$ is an extension of $\psi$ to $\widetilde{\Omega} \times(0, T)$ by 0 and is then extended periodically to $\mathbb{R}^{N} \times(0, T)$. Now we assume to have a solution $\left(n^{\varepsilon}, u^{\varepsilon}\right)$ of $(3.28)$ satisfying all of the properties listed in Theorem 3.5 and (3.26) is replaced by

$$
\begin{aligned}
\int_{\Omega} n^{\varepsilon} \frac{1}{2}\left|u^{\varepsilon}\right|^{2} & +\frac{a}{\gamma-1}\left(n^{\varepsilon}\right)^{\gamma} d x+\int_{0}^{t} d s \int_{\tilde{\Omega}} \mu\left|D u^{\varepsilon}\right|^{2}+\xi\left(\operatorname{div} u^{\varepsilon}\right)^{2}+n^{\varepsilon}\left|u^{\varepsilon}\right|^{2}+\frac{1}{\varepsilon} p u^{\varepsilon} d x \\
& \leq \int_{\tilde{\Omega}} \frac{1}{2} \frac{\left|\widetilde{j}_{0}\right|^{2}}{\widetilde{n}_{0}}+\frac{a}{\gamma-1} \tilde{n}_{0}^{\gamma} d x+\int_{0}^{t} d s \int_{\tilde{\Omega}} n^{\varepsilon} u^{\varepsilon} \nabla \tilde{\psi} d x
\end{aligned}
$$

Of course, $\left.n^{\varepsilon}\right|_{t=0}=\widetilde{n}_{0},\left.n^{\varepsilon} u^{\varepsilon}\right|_{t=0}=\widetilde{j}_{0}$, and $\left(\widetilde{n}_{0}, \widetilde{j}_{0}\right)$ are periodic extensions of $\left(n_{0}, j_{0}\right)$ by 0 . The inequality $(3.29)$ yields bounds on $\left(n^{\varepsilon}\right)^{\gamma}$ in $L^{\infty}\left(0, T ; L^{1}(\widetilde{\Omega})\right)$, on $n^{\varepsilon}\left|u^{\varepsilon}\right|^{2}$ in $L(\widetilde{\Omega} \times(0, T))$, on $u^{\varepsilon}$ in $L^{2}\left(0, T ; H^{1}(\widetilde{\Omega})\right)$, and on $\frac{1}{\varepsilon} p u^{\varepsilon}$ in $L^{1}(\widetilde{\Omega} \times(0, T))$ uniformly in $\varepsilon$. In addition, Theorem 3.3 yields a bound on $\left(n^{\varepsilon}\right)$ in $L^{p}(K \times(0, T))$ for any compact set $K \subset \Omega$, where $p=\gamma+\frac{2}{N}-1$. Extracting subsequences if necessary, we may assume that $n^{\varepsilon}$ converges weakly in $L^{r}\left(0, T ; L^{\gamma}(\widetilde{\Omega})\right)$ for all $r \in(1, \infty)$ as $\varepsilon$ goes to 0 to some $n \in L^{\infty}\left(0, T ; L^{\gamma}(\widetilde{\Omega}) \cap L^{p}(K \times(0, T))\right.$ for any compact set $K \subset \Omega$, and we may assume that $u^{\varepsilon}$ converges weakly in $L^{2}\left(0, T ; H^{1}(\widetilde{\Omega})\right)$ as $\varepsilon$ goes to 0 to some $u$. In addition, since $\frac{1}{\sqrt{\varepsilon}} \sqrt{p} u^{\varepsilon}$ is bounded in $L^{2}(\widetilde{\Omega} \times(0, T)), \sqrt{p} u^{\varepsilon}$ converges to 0 in $L^{2}(\widetilde{\Omega} \times(0, T))$ and thus $\widetilde{p} u \equiv 0$ in $\widetilde{\Omega} \times(0, T)$. Therefore $u=0$ a.e. in $(\widetilde{\Omega}-\Omega) \times(0, T)$. Since $\Omega$ is smooth, this implies that $u \in L^{2}\left(0, T ; H_{0}^{1}\right)$.

Finally, in order to conclude, we simply observe that we can apply Theorem 3.2 to the 
sequences $\left(n^{\varepsilon}, u^{\varepsilon}\right)$. In fact we (an apply part (1) and part (2) of Theorem 3.2. Finally from the energy inequality (3.26) we get (3.27). Now we have to show how to construct a solution for (3.28); for this we can follow [15].

\section{Existence for the Coupled Navier-Stokes-Poisson equation.}

4.1. Local existence. We recall we are studying the following problem:

$$
\left\{\begin{array}{l}
\frac{\partial n}{\partial t}+\operatorname{div}(n u)=0 \\
\frac{\partial n u}{\partial t}+\operatorname{div}(n u \otimes u)+\nabla\left(a n^{\gamma}\right)-\mu \Delta u-\xi \nabla \operatorname{div} u=n \nabla \phi-n u \\
\Delta \phi=n-b(x)
\end{array}\right.
$$

with the initial conditions (2.5). the boundary condition (2.6), and the hypotheses (A.1), (A.2), (A.3), (A.4), (A.5). We wish to solve the previous problem by using a fixed point argument on the potential 0 ; namely, we consider

$$
\begin{aligned}
& \frac{\partial n}{\partial t}+\operatorname{div}(n u)=0 \\
& \frac{\partial m u}{\partial t}+\operatorname{div}(n u \& u)+\nabla\left(a m^{\gamma}\right)-\mu \Delta u-\xi \nabla \operatorname{div} u=n \nabla \psi-n u \\
& \Delta \phi=n-b(x)
\end{aligned}
$$

with the potential $\psi$ given. We assume the initial condition (2.5), the boundary conditions (2.6), the hypotheses (A.1). (A.2), (A.3), (A.4), (A.5), and the following hypothesis for all $T>0$ :

(A.6)

$$
\begin{array}{lr}
\psi \in L^{1}\left(0 . T: W^{1.2 \gamma /(\gamma-1)}\right) & \text { if } N \geq 2, \\
\psi \in L^{1}\left(0, T: W^{1.2}\right) & \text { if } N=2 \text { and } \gamma>2, \\
\psi \in L^{1}\left(0 . T: W^{1.3 / 2}\right) & \text { if } N=3 \text { and } \gamma>6 .
\end{array}
$$

We now define the mapping for which a fixed point will yield a solution of (4.1). Let us denote by

$$
\begin{aligned}
& F\left(0, T_{1} ; R\right)=\left\{\phi \in L^{1}\left(0 . T_{1}, W^{1,2 \gamma / \gamma-1}(\Omega)\right) \mid\left\|\phi-\phi_{0}\right\|_{L^{1}\left(0 . T_{1} \cdot W^{1.2 \gamma / \gamma-1}(\Omega)\right)} \leq R\right\} \\
& \text { if } N \geq 8 \text { and } \gamma>N / 2 \text { or } 4 \leq N<8 \text { and } \gamma>5 N /(N+2) \text { or } N=3 \text { and } 3 \leq \gamma \leq 6, \\
& F\left(0, T_{1} ; R\right)=\left\{\phi \in L^{1}\left(0, T_{1}, W^{1,2}(\Omega)\right) \mid\left\|\phi-\phi_{0}\right\|_{L^{1}\left(0 . T_{1} \cdot W^{1.2}(\Omega)\right)} \leq R\right\} \\
& \text { if } N=2 \text { and } \gamma>2 \text {, and } \\
& F\left(0, T_{1} ; R\right)=\left\{\phi \in L^{1}\left(0, T_{1}, W^{1.3 / 2}(\Omega)\right) \mid\left\|\phi-\phi_{0}\right\|_{L^{1}\left(0 . T_{1}, W^{1.3 / 2}(\Omega)\right)} \leq R\right\} \\
& \text { if } N=3 \text { and } \gamma>6 .
\end{aligned}
$$

We define a map from $F\left(0 . T_{1}: R\right)$ into itself as follows:

$$
A: F\left(0, T_{1} ; R\right) \longrightarrow F\left(0, T_{1} ; R\right)
$$


and

$$
A \psi=\phi \quad \text { if and only if } \quad \Delta \phi=n-b(x),
$$

where $(n, u)$ is the weak solution to the Navier-Stokes equation (4.2), (4.3) with a given potential $\psi$. The map $A$ is well-posed, since from the previous section we have the existence of weak solutions to (4.2), (4.3) in any arbitrary time interval $[0, T]$. Now we show the existence of a fixed point which will give a local weak solution to (4.1) with the regularity defined in (2.7), (2.8), (2.9), and (2.10).

Theorem 4.1. Suppose that (A.1), (A.2), (A.3), (A.4), (A.5), (A.6) hold. Then if $N \geq 8$ and $\gamma \geq N / 2$ or $3<N<8$ and $\gamma>5 N /(N+2)$ or $N=3$ and $3<\gamma \leq 6$, there exists $T_{1}>0$ such that the map $A$ is continuous and compact.

Proof. From Theorem 3.5 we have that $n$ is bounded in $L^{\infty}\left(0, T ; L^{\gamma}(\Omega)\right)$, while $j=n u$ is bounded in $L^{\infty}\left(0, T ; L^{2 \gamma / \gamma+1}(\Omega)\right)$. More precisely, there exists a constant $c(R)>0$ depending only on the initial data such that

$$
\left\|n-n_{0}\right\|_{L^{\infty}\left(0, T ; L^{\gamma}(\Omega)\right)} \leq c(R) \quad\|j\|_{L^{\infty}\left(0, T ; L^{2 \gamma /(\gamma+1)}(\Omega)\right)} \leq c(R) .
$$

From the Poisson equation (4.4) we get in the sense of distribution

$$
\operatorname{div}\left[\nabla \phi_{t}\right]=-\operatorname{divj}
$$

and applying the elliptic regularity Theorem 2.2 we have

$$
\begin{aligned}
\left\|A \psi(\cdot, t)-\phi_{0}(\cdot)\right\|_{L^{s}(\Omega)} & \leq\left\|A \psi(\cdot, t)-\phi_{0}(\cdot)\right\|_{W^{1,2 \gamma /(\gamma+1)}(\Omega)} \\
& =\left\|\phi(\cdot, t)-\phi_{0}(\cdot)\right\|_{W^{1,2 \gamma /(\gamma+1)}(\Omega)} \\
& \leq t\|j\|_{L^{\infty}\left(0, T ; L^{2 \gamma /(\gamma+1)}(\Omega)\right)} \leq t c(R),
\end{aligned}
$$

where $s$ is the Sobolev exponent of $\frac{2 \gamma}{\gamma+1}$. We can also observe that

$$
\Delta \phi(\cdot, t)-\Delta \phi(\cdot, 0)=n(\cdot, t)-n_{0}(\cdot),
$$

and so, applying Theorem 2.2 again we get

$$
\left\|A \psi(\cdot, t)-\phi_{0}(\cdot)\right\|_{W^{2, \gamma}(\Omega)} \leq\left\|n(\cdot, t)-n_{0}(\cdot)\right\|_{L^{\gamma}(\Omega)} \leq c(R) .
$$

Now we apply (2.2) of Theorem 2.3 to have

$$
\begin{aligned}
\left\|A \psi(\cdot, t)-\phi_{0}(\cdot)\right\|_{W^{1,2 \gamma /(\gamma-1)}(\Omega)} & \leq c\left\|\phi(\cdot, t)-\phi_{0}(\cdot)\right\|_{W^{2, \gamma}(\Omega)}^{1 / 2}\left\|\phi(\cdot, t)-\phi_{0}(\cdot)\right\|_{L^{q}(\Omega)}^{1 / 2} \\
& \leq c(R) t^{1 / 2}
\end{aligned}
$$

where $q=\frac{\gamma}{\gamma-2}$ and provided that $q<s$, which is true if $N \geq 8$ and $\gamma \geq N / 2$ or $3<N<8$ and $\gamma>5 N /(N+2)$. We have

$$
\left\|A \psi-\phi_{0}\right\|_{L^{1}\left(0, T_{1}, W^{1,2 \gamma /(\gamma-1)}(\Omega)\right)} \leq c(R) T_{1}^{3 / 2} .
$$

Now taking, for instance,

$$
T_{1}=\frac{1}{2}\left(\frac{R}{c(R)}\right)^{2 / 3}
$$


we get that $A$ is continuous from $F\left(0, T_{1} ; R\right)$ into itself. Finally, we have to show that $A$ is a compact map. It is sufficient to see that the range of $A$ is relatively compact. Applying Theorem 2.2 again we have, taking $\psi \in F\left(0, T_{1} ; R\right)$,

$$
\|A \psi(\cdot, t)-A \psi(\cdot, s)\|_{W^{1,2 \gamma /(\gamma+1)}} \leq c|t-s|\|j\|_{L^{\infty}\left(0, T ; L^{2 \gamma /(\gamma+1)(\Omega))}\right.} \leq c(R)|t-s|,
$$

so $A F\left(0, T_{1} ; R\right) \in C^{1 / 2}\left(0, T, W^{1.2 \gamma /(\gamma-1)}(\Omega)\right)$. Moreover, since $A \psi(\cdot, t)-\phi_{0}(\cdot) \in$ $W_{0}^{1, \gamma}(\Omega) \cap W^{2, \gamma}(\Omega)$, by using the hypotheses on $\gamma$ we get $A F\left(0, T_{1} ; R\right)$, for any fixed $t$, is relatively compact in $W^{1,2 \gamma /(\gamma-1)}(\Omega)$. In this way, all the hypotheses of Theorem 2.1 are fullfilled and we get, finally, the compactness of the map $A$.

Now we investigate what happens in the other cases.

Theorem 4.2. Suppose that (A.1), (A.2), (A.3), (A.4). (A.5), (A.6) hold. Then if $N=3$ and $\gamma=3$ or $\gamma \geq 6$, there exists $T_{1}>0$ such that the map $A$ is continuous and compact.

Proof. If $\gamma>6$ by hypothesis (A.6), we can take $\psi \in L^{1}\left(0, T_{1}, W^{1,3 / 2}(\Omega)\right)$, and so by Theorem 2.2 we have

$$
\begin{aligned}
\left\|A \psi(\cdot, t)-\phi_{0}(\cdot)\right\|_{W^{1.3 / 2}} & \leq\left\|A \psi(\cdot, t)-A \phi_{0}(\cdot)\right\|_{W^{1.2 \gamma /(\gamma+1)}} \leq t\|j\|_{L^{\infty}\left(0, T: L^{2 \gamma /(\gamma+1)}(\Omega)\right)} \\
& \leq t(R),
\end{aligned}
$$

and, moreover,

$$
\left\|A \psi-\phi_{0}\right\|_{L^{1}\left(0, T_{1} \cdot W^{1.3 / 2}(\Omega)\right)} \leq c(R) T_{1}^{2} .
$$

When $\gamma=3$, by applying the Sobolev embeddings theorem and the elliptic regularity theorem, we get

$$
\left\|\phi(\cdot, t)-\phi_{0}(\cdot)\right\|_{L^{*}(\Omega)} \leq t\|j\|_{L^{\infty}\left(0, T, L^{2 \gamma /(\gamma+1)}(\Omega)\right)},
$$

where $s$ is the Sobolev exponent of $\frac{2 \gamma}{\gamma+1}$. Using the interpolation inequality (2.3) of Theorem 2.3 we get

$$
\left\|\nabla \phi(\cdot, t)-\nabla \phi_{0}(\cdot)\right\|_{L^{\gamma}(\Omega)} \leq c\left\|\phi(\cdot, t)-\phi_{0}(\cdot)\right\|_{W^{2, \gamma}(\Omega)}^{1 / 2}\left\|\phi(\cdot, t)-\phi_{0}(\cdot)\right\|_{L^{\gamma}(\Omega)}^{1 / 2} .
$$

If $s \geq \gamma$ we get

$$
\begin{aligned}
\left\|\nabla \phi(\cdot, t)-\nabla \phi_{0}(\cdot)\right\|_{L^{\gamma}(\Omega)} & \leq c(R)^{1 / 2}\left\|\phi(\cdot, t)-\phi_{0}(\cdot)\right\|_{L^{s}(\Omega)} \\
& \leq c(R) t^{1 / 2}\|j\|_{L^{\infty}\left(0 . T, L^{2 \gamma /(\gamma+1)}(\Omega)\right)} \leq c(R) t^{1 / 2} .
\end{aligned}
$$

Now provided $\gamma>\frac{2 \gamma}{\gamma-1}$ and choosing $t$ sufficiently small, we get as in the previous theorem our result. We have only to verify that $\frac{2 \gamma}{\gamma-1}<\gamma<s$, and this is the case if $\gamma=3$ (actually this is the only case). As in Theorem 4.1 we get the compactness of A.

Finally we investigate the case $N=2$.

Theorem 4.3. Suppose that (A.1), (A.2), (A.3), (A.4), (A.5), (A.6) hold. If $N=2$ and $\gamma>2$, there exists $T_{1}>0$ such that the map $A$ is continuous and compact.

Proof. In the case $N=2$ and $\gamma>2$, we follow the same argument of Theorem 4.1 with the difference that, in this case, $s=2 \gamma$ and $q=\frac{\gamma-1}{\gamma}$ and since $\gamma>2$ we have $q<s$. 
The compactness of $A$ follows by using Theorem 2.1 in the same way as in Theorem 4.1 .

Now we can conclude with the main result in this section:

Theorem 4.4. Suppose that (A.1), (A.2), (A.3), (A.4), (A.5), (A.6) hold. Then if $N \geq 8$ and $\gamma>N / 2$ or $4 \leq N<8$ and $\gamma>5 N /(N+2)$ or $N=3$ and $\gamma \geq 3$ or $N=2$ and $\gamma>2$, there exists a local solution of system (2.4).

Proof. The theorem is a consequence of Theorem 3.5 and Schauder's fixed point Theorem ([21], pg. 84). In fact, by Theorem 3.5, we get the existence of $n$ and $u$ in an arbitrary time interval for (4.2), (4.3). Using Theorem 4.1, Theorem 4.2, and Theorem 4.3, we can apply Schauder's fixed point Theorem ([21], pg. 84) to the map $A$, getting a fixed point $\phi$ for the map $A$. Namely, there exist $\phi \in F\left(0, T_{1} ; R\right)$ such that $\Delta \phi=n-b(x)$; that is our solution to the Cauchy problem.

4.2. Global existence. From Theorem 4.4 we have the existence of a local weak solution $(n, u, \phi)$ in a time interval $\left[0, T^{*}\right)$, where $T^{*}$ is the maximal time of existence. We want to prove that we can extend the weak solution of the system (4.1) to the whole interval $\left[0, T^{*}\right]$. From the energy estimates follows

Theorem 4.5. Let $(n, u, \phi)$ be a local weak solution of system (4.1); then $j=n u$ is equibounded in $L^{\infty}\left(0, T^{*} ; L^{2 \gamma /(\gamma+1)}(\Omega)\right)$.

Proof. Multiplying Eq. (4.3) by $u$ and integrating with respect to $x$ and remembering the boundary conditions on $u$, we deduce that we have for some $\nu>0$

$$
\begin{aligned}
& \frac{d}{d t} \int_{\Omega}\left\{n \frac{|u|^{2}}{2}+\frac{a}{\gamma-1} n^{\gamma}\right\} d x+\nu \int_{\Omega}|D u|^{2} d x+\frac{3 \gamma-1}{2 \gamma} \int_{\Omega} n \frac{|u|^{2}}{2} d x \\
& \leq \frac{\gamma+1}{4 \gamma} \int_{\Omega} n^{\gamma} d x+\frac{\gamma-1}{4 \gamma}\|\nabla \phi(\cdot, t)\|_{L^{2 \gamma /(\gamma-1)(\Omega)} .}
\end{aligned}
$$

Using Gronwall's lemma we get for any $t \in\left[0, T^{*}\right)$

$$
\int_{\Omega}\left\{n \frac{|u|^{2}}{2}+\frac{a}{\gamma-1} n^{\gamma}\right\} d x \leq C_{1}+C_{2} \int_{0}^{T^{*}}\|\nabla \phi(\cdot, s)\|_{L^{2 \gamma / \gamma-1}(\Omega)} d s
$$

and

$$
\begin{aligned}
\|j(\cdot, t)\|_{L^{2 \gamma / \gamma+1}(\Omega)} & \leq\|n(\cdot, t)\|_{L^{\gamma}(\Omega)}^{\gamma / 2}\left\|n u^{2}(\cdot, t)\right\|_{L^{1}(\Omega)}^{1 / 2} \\
& \leq C_{1}+C_{2} \int_{0}^{T^{*}}\|\nabla \phi(\cdot, s)\|_{L^{2 \gamma / \gamma-1}(\Omega)} d s .
\end{aligned}
$$

By Theorem 2.2 and the previous estimates we get (from (4.1))

$$
\begin{aligned}
\frac{d}{d t}\|\nabla \phi(\cdot, t)\|_{L^{2 \gamma /(\gamma+1)}(\Omega)} & \leq\left\|\nabla \phi_{t}(\cdot, t)\right\|_{L^{2 \gamma / \gamma+1}(\Omega)} \leq c\|j(\cdot, t)\|_{L^{2 \gamma /(\gamma+1)}(\Omega)} \\
& \leq C_{1}+C_{2} \sup _{t \in\left[0, T^{*}\right]}\|\nabla \phi(\cdot, t)\|_{L^{2 \gamma / \gamma-1}(\Omega)} .
\end{aligned}
$$

By Gronwall's lemma there exists $M>0$ such that

$$
\sup _{t \in\left[0, T^{*}\right]}\|\nabla \phi(\cdot, t)\|_{L^{2 \gamma / \gamma+1}(\Omega)} \leq M
$$


from which follows the equibound on $j=n u$.

Corollary 4.6. Suppose $T^{*}$ is the maximal time of existence of the solutions of system (4.1) and that the hypotheses of Theorem 4.4 hold; then

$$
\begin{array}{lr}
\phi \in C^{1 / 2}\left(0, T_{1}: W^{1.2 \gamma /(\gamma-1)}(\Omega)\right) & \text { if } N \geq 2, \\
\phi \in C^{1 / 2}\left(0 . T_{1}: W^{1.2}(\Omega)\right) & \text { if } N=2 \text { and } \gamma>2, \\
\phi \in \operatorname{Lip}\left(0, T_{1} ; W^{1.3 / 2}(\Omega)\right) & \text { if } N=3 \text { and } \gamma>6
\end{array}
$$

moreover, $\phi$ is equicontinuous in time.

Proof. Combining the equation of system (4.1) and Theorem 2.2, we have

$$
\left\|\phi(\cdot, t)-\phi_{0}(\cdot, s)\right\|_{W^{1,2 \gamma /(\gamma+1)}(\Omega)} \leq|t-s|\|j\|_{L^{x}\left(0, T^{*}: L^{2 \gamma /(\gamma+1)(\Omega))}\right.} ;
$$

by Theorem 4.5 and the values of $\gamma$ we get the equicontinuity in time of $\phi$.

By the previous results and by using standard continuation methods, we obtain the following globally in time existence theorem.

Theorem 4.7. Assume that $N \geq 8$ and $\gamma>N / 2$ or $4 \leq N<8$ and $\gamma>5 N /(N+2)$ or $N=3$ and $\gamma \geq 3$ or $N=2$ and $\gamma>2$. If (A.1), (A.2), (A.3), (A.4), and (A.5) hold, then the system (2.4) has a global weak solution satisfying (2.7), (2.8), (2.9), (2.10).

\section{REFERENCES}

[1] R. A. Adams. Sobolev Spaces, Academic Press, 1975

[2] A. M. Anile, An extended thermodynamic framework for the hydrodynamical modeling of semiconductors, Pitman Research Notes In Mathematics Series, 340. 1995, pp. 3-41.

[3] A. M. Anile and S. Pennisi. Extended thermodynamics of the Blotekjaer hydrodynamical model for semiconductors. Continuum Mech. Therm., 4, 1992, pp. 187-193.

[4] H. Brezis, Functional Analysis - Theory and Applications, Ligouri Editore.

[5] R. Coifman, R. Rochberg, and G. Weiss, Ann. Math., 103, 1975, pp. 611-635.

[6] C. Cercignani, The Boltzmann equation and its applications. Applied Mathematical Sciences, 67. Springer-Verlag, New York.

[7] C. Cercignani, R. Illner, and M. Pulvirenti, The mathematical theory of dilute gases, Applied Mathematical Sciences, 106, 1994. Springer-Verlag, New York.

[8] B. Desjardins, Weak solutions on the compressible isentropic Navier-Stokes equations. Appl. Math. Lett., 12, 1999, no. 7. 107 111.

[9] L. C. Evans, Partial Differential Equations, volume 19 of Graduate Studies in Mathematics. American Mathematical Society.

[10] V. I. Gerasimenko and D. Ya. Petrina, The Boltzmann-Grad limit theorem (Russian), Dokl. Akad. Nauk Ukrain. SSR Ser. A. 1989, no. 11, 12-16.

[11] D. Gilbarg and N. S. Trudinger. Elliptic Partial Differential Equations of Second Order, volume 224 of Grundlehren der matematischen Wissenschaften, Springer-Verlag, 1977.

[12] C. D. Levermore, Moment closure hierarchies for kinetic theories, J. Statist. Phys., 83, 1996, no. 5-6, 1021-1065.

[13] C. D. Levermore, Entropy-based moment closures for kinetic equations. Proceedings of the International Conference on Latest Developments and Fundamental Advances in Radiative Transfer (Los Angeles, CA, 1996), Transport Theory Statist. Phys., 26, 1997, no. 4-5, 591-606.

[14] P. L. Lions, Mathematical Topics in Fluid Mechanics, Vol. 1, Incompressible Models, Clarendon Press, Oxford Science Publications. Oxford, 1996

[15] P. L. Lions, Mathematical Topics in Fluid Mechanics, Vol. 2, Compressible Models. Clarendon Press, Oxford Science Publications, Oxford, 1998

[16] P. Marcati and R. Natalini, Weak solutions to a hydrodynamic model for semiconductors and relaxation to the drift-diffusion equation, Arch. Rational Mech. Anal., 129, 1995, 129-145. 
[17] P. Marcati and R. Natalini, Weak solutions to a hydrodynamic model for semiconductors: the Cauchy problem, Proc. Roy. Soc. Edinburgh Sect. A, 125 (1995), no. 1, 115-131.

[18] P. A. Markowich, The Steady-State Semiconductor Device Equations, Springer, New York, 1986.

[19] P. A. Markowich, C. Ringhofer, and C. Schmeiser, Semiconductor Equations, 1990, Springer-Verlag, Wien, New York.

[20] N. G. Meyers, An $L^{p}$ estimate for the gradient of solutions of the second order elliptic divergence equations, Annali della Scuola Normale Superiore, 1963, 189-205.

[21] L. Nirenberg, Functional Analysis, Courant Institute of Math. Sciences, New York, 1984.

[22] L. C. Piccinini, G. Stampacchia, and G. Vidossich, Ordinary Differential Equations in $R^{N}$, Applied Mathematical Sciences 39, Springer-Verlag, New York, 1984.

[23] R. Temam, Navier-Stokes Equations, North-Holland, Amsterdam, 1977.

[24] S. Ukai, The Boltzmann-Grad limit and Cauchy-Kovalevskaya theorem, Recent topics in mathematics moving toward science and engineering. Japan J. Indust. Appl. Math., 18, 2001, no. 2, 383-392. 